\title{
Clean Your Hands May 5, 2017: Fight Antibiotic Resistance-It's in Your Hands
}

\author{
Ermira Tartari, MS; ${ }^{1,2}$ Daniela Pires, MD; ${ }^{1,3}$ Didier Pittet, MD, MS ${ }^{1}$
}

Preventing healthcare-associated infections and reducing their avoidable impact on health systems is critical today to make facilities safer for patients worldwide. ${ }^{1}$ In addition, the increasing public health burden of antimicrobial resistance (AMR) urges action. ${ }^{2}$ Stronger political commitment in reducing AMR was highlighted at the last United Nations General Assembly in September 2016 in New York.

Hand hygiene is at the center of effective infection prevention and control (IPC) to combat AMR spread. ${ }^{3}$ The World Health Organization (WHO) recently issued guidelines on the Core Components of effective IPC program. ${ }^{1}$ Their implementation will help create strong, resilient health systems in all settings. The guidelines include the application of a multimodal strategy: achieving system change (infrastructure and resources), raising awareness, increasing education and training, monitoring and timely feedback, and creating a patient safety culture that includes visibly committed leadership. This approach improves hand hygiene, reduces infections, and saves lives. ${ }^{4}$ Therefore, on the May 5, 2017, Global Annual Hand Hygiene Day, WHO urges policy makers, top-level managers, IPC specialists, and other health professionals to focus on the fight against AMR spread by building everstronger hand hygiene and IPC programs (Table 1).

TABLE 1. May 5, 2017 Key World Health Organization Campaign Messages

Health workers: "Clean your hands at the right times and stop the spread of antibiotic resistance."

Hospital Chief Executive Officers and Administrators: "Lead a yearround infection prevention and control program to protect your patients from resistant infections."

Policy makers: "Stop antibiotic resistance spread by making infection prevention and hand hygiene a national policy priority."

IPC leaders: "Implement WHO's Core Components for infection prevention, including hand hygiene, to combat antibiotic resistance."
We encourage health facilities worldwide to endorse the May 5, 2017, WHO campaign ${ }^{5}$ and to further improve hand hygiene, fight antibiotic resistance, and commit to progressing toward adherence to all core components of the IPC program.

Let's fight antibiotic resistance together; it's in our hands.

\section{ACKNOWLEDGMENTS}

Financial support: No financial support was provided relevant to this article. Potential conflicts of interest: All authors report no conflicts of interest relevant to this article.

\section{REFERENCES}

1. Guidelines on core components of infection prevention and control programmes at the national and acute health care facility level. World Health Organization website. http://apps.who.int/ iris/handle/10665/251730. Published 2016. Accessed January 30, 2017.

2. Global action plan on antimicrobial resistance. World Health Organization website. http://apps.who.int/iris/handle/10665/193736. Published 2015. Accessed January 30, 2017.

3. WHO guidelines on hand hygiene in health care: first global patient safety challenge: clean care is safer care. Geneva, Switzerland. World Health Organization website. http://apps. who.int/iris/bitstream/10665/44102/1/9789241597906_eng.pdf. Published 2009. Accessed January 30, 2017.

4. Luangasanatip $\mathrm{N}$, Hongsuwan $\mathrm{M}$, Limmathurotsakul $\mathrm{D}$, et al. Comparative efficacy of interventions to promote hand hygiene in hospital: systematic review and network meta-analysis. BMJ 2015;351:h3728.

5. Save lives: clean your hands. WHO's global annual campaign advocacy toolkit. World Health Organization website. http:// www.who.int/gpsc/5may_advocacy-toolkit.pdf?ua=1. Accessed January 30, 2017.

Affiliations: 1. Infection Control Programme and WHO Collaborating Centre on Patient Safety-Infection Control \& Improving Practices, University of Geneva Hospitals and Faculty of Medicine, Geneva, Switzerland; 2. Infection Control Unit, Mater Dei Hospital, Msida, Malta; 3. Department of Infectious Diseases, Centro Hospitalar Lisboa Norte and Faculdade de Medicina da Universidade de Lisboa, Lisbon, Portugal.

Received February 6, 2017; accepted February 12, 2017

(c) 2017 by The Society for Healthcare Epidemiology of America. All rights reserved. 0899-823X/2017/3804-0022. DOI: 10.1017/ice.2017.43 